\title{
A Versatile Microfluidic Chip for Millisecond Time-Scale Kinetic Studies by Electrospray Mass Spectrometry
}

\author{
Tamanna Rob and Derek J. Wilson \\ Center for Research in Mass Spectrometry, Department of Chemistry, York University, Toronto, Ontario, Canada
}

An electrospray coupled microfluidic reactor for the measurement of millisecond time-scale, solution phase kinetics is introduced. The device incorporates a simple two-channel design that is etched into polymethyl methacrylate (PMMA) by laser ablation. The outlet of the device is laser cut to a sharp tip, facilitating low dead volume 'on chip' electrospray. Fabrication is fast, straightforward and highly reproducible, supporting rapid prototyping and large-scale reproduction. Device performance is characterized using a cytochrome $c$ unfolding reaction. Unfolding processes with rates in excess of $30 \mathrm{~s}^{-1}$ are easily measured, including the appearance of a 'native-like' intermediate that is maximally populated $180 \mathrm{~ms}$ post reaction initiation. To extract reliable rates from the data, a theoretical framework for the analysis of kinetics acquired under square-channel laminar flow is introduced. (J Am Soc Mass Spectrom 2009, 20, 124-130) (C) 2009 Published by Elsevier Inc. on behalf of American Society for Mass Spectrometry

$\mathrm{M}$ icrofluidics involves the fabrication of microscale features, usually in glass, silicon, or polymer substrates, to enable the precise handling of small-volume liquid samples. Micro-scale liquid handling facilitates efficient mass and heat transfer, reduced sample consumption, and high throughput via parallelization while minimizing sample loss and cross contamination. There is therefore widespread interest in the development of modular 'lab on a chip' devices with integrated sample preparation, separation and reactor functionality [1].

ESI coupled microfluidic devices (ECMDs) have been devised for numerous applications, particularly in the area of proteomics [2]. The complementary advantages of microfluidic liquid handling and ESI-MS based analysis make these devices a natural answer to the challenges of proteomic studies. One 'underrepresented' application is rapid microfluidic mixing for time-resolved studies by ESI-MS. Time-resolved ESI-MS (TR ESI-MS) is a powerful approach for the characterization of shortlived, 'transient' intermediates in biochemical reactions [3-10], which are critical to the biological activity of proteins, from enzymatic reactions $[7,11]$ to protein folding $[12,13]$.

In order for TR ESI-MS to present a viable alternative to optical time-resolved methods, it must be possible to acquire complete reaction time-courses in a single experiment. This motivated the development of 'Stopped Flow ESI-MS' by Kolakowski and Konermann in 2000 [9, 10]. In 2003, Wilson and Konermann introduced a

Address reprint requests to Dr. D. J. Wilson, Department of Chemistry, York University, Toronto, ON M3J 1P3, Canada. E-mail: dkwilson@yorku.ca http://www.yorku.ca/dkwilson/ continuous flow capillary mixer for TR ESI-MS that facilitated full time-course acquisitions on time-scales comparable to optical stopped flow experiments [3]. This approach has enabled numerous investigations $[3-5,14-17]$, but the concentric capillary design is limiting in terms of reproducibility of fabrication and is not well suited to the inclusion of directly coupled sample preparation or separation steps.

This work introduces a new microfluidic device for TR ESI-MS. The device incorporates an internal capillary mixer that is similar to the one in reference [3], but formed by laser ablation with greatly improved reproducibility. Device performance is characterized by monitoring acid induced cytochrome $c$ unfolding kinetics. To enable the extraction of reliable rate constants, a theoretical framework for the analysis of kinetic data acquired under laminar flow in a square channel is devised.

\section{Experimental}

\section{Chemicals and Supplies}

Horse heart cytochrome $c$ was purchased from Sigma (St. Louis, MO). Ultrapure water was generated inhouse on a Millipore Milli-Q Advantage A10 system. High purity acetic acid $(99.7+\%)$ was purchased from Sigma. Blank PMMA substrates with dimension $(l=8.9$ $\mathrm{cm}, w=3.8 \mathrm{~cm}, h=0.3 \mathrm{~cm}$ ) were supplied by Professional Plastics (Fullerton, CA). Polyimide coated glass capillaries (O.D. $153 \mu \mathrm{m}$, i.d. $75 \mu \mathrm{m}$ ) were supplied by Polymicro Technologies (Phoenix, AZ). Metal capillaries (o.d. $400 \mu \mathrm{m}$, i.d. $200 \mu \mathrm{m})$ were from Small Parts, Inc. (Miramar, FL). PTFE tubing (o.d. 1/16 in., i.d. $400 \mu \mathrm{m}$ ) 
was purchased from McMaster Plastics (Scarborough, ON). Standard 1/16 in. fittings were supplied by Upchurch (Oak Harbor, WA).

\section{Microfluidic Device Fabrication}

The microfluidic reactor was fabricated using a VersaLaser engraving device (Universal Laser, Scottsdale, AZ). The microfluidic channel design was generated in CorelDraw X3 (Corel, Ottawa, ON). Laser power and mode were differentiated by color $($ red $=$ vector mode cutting, blue $=$ vector mode marking, black $=$ raster mode). Microfluidic channel widths were adjusted to $155 \mu \mathrm{m} \pm 2$ by slightly defocusing the laser. The channels were engraved into blank PMMA substrate to a depth of $155 \mu \mathrm{m} \pm 2$ using two vector marking runs at $2.2 \mathrm{~J} / \mathrm{cm}^{2}$. The distal end of the device was cut to a sharp tip using vector cutting mode at $4.6 \mathrm{~J} / \mathrm{cm}^{2}$. At the proximal end of the device, channels were connected to external 1/16 in. Upchurch fittings by using thin-walled metal capillaries as 'step up' unions. The metal capillaries were melted into the channel inlets at one end and pressure fit into 1/16 in. o.d. PTFE tubing at the other. A second blank PMMA substrate was used as the cover. The chip and cover were placed in a vice $(\sim 5 \mathrm{MPa})$ and thermally bonded in a convection oven $\left(120^{\circ} \mathrm{C}\right.$ for 40 min). Fluids were introduced into the device using Harvard 11+ infusion pumps (Holliston, MA). Typical flow rates were $7.0 \mu \mathrm{L} / \mathrm{min}$ for both reactants. A picture of the device is shown in Figure 1a.

The main microfluidic channel was sized to accommodate a $153 \mu \mathrm{m}$ o.d. fused silica 'mixing capillary'. The distal end of the capillary was sealed with molten fused silica by cutting at high laser power. Vector marking mode $\left(1.1 \mathrm{~J} / \mathrm{cm}^{2}\right)$ was used to cut a notch in the capillary $2 \mathrm{~mm}$ from the sealed end. A microscopic view of the mixer inside the main microfluidic channel is provided in Figure 1b. Fluid passing through the capillary (blue arrow) exits through the notch and is forced into the narrow space between the capillary and the channel walls. Here it mixes with fluid that has passed through the channel around the inner capillary. The observed reaction time is adjusted by changing the position of the mixing capillary within the channel. Early reaction times are observed when the mixer is positioned close to the ESI outlet. Steadily distancing the mixer from the ESI outlet yields mass spectra corresponding to steadily later reaction times.

\section{TR ESI-MS Experiments}

Time-resolved mass spectrometry was carried out on a QStar Elite Q-TOF instrument (MDS Analytical Technologies, Concord, ON) with an in-house built source assembly. The source recognition logic was circumvented using a custom switch. The instrument was operated in positive ion mode with source voltage $+5500 \mathrm{~V}$ and moderate declustering conditions (declustering potential $(\mathrm{DP})=60-90 \mathrm{~V}$, focusing ring $(\mathrm{FP})=$

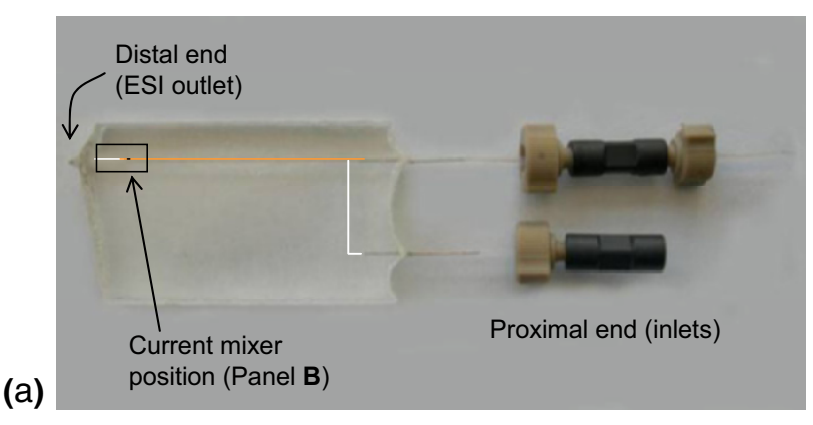

(b)

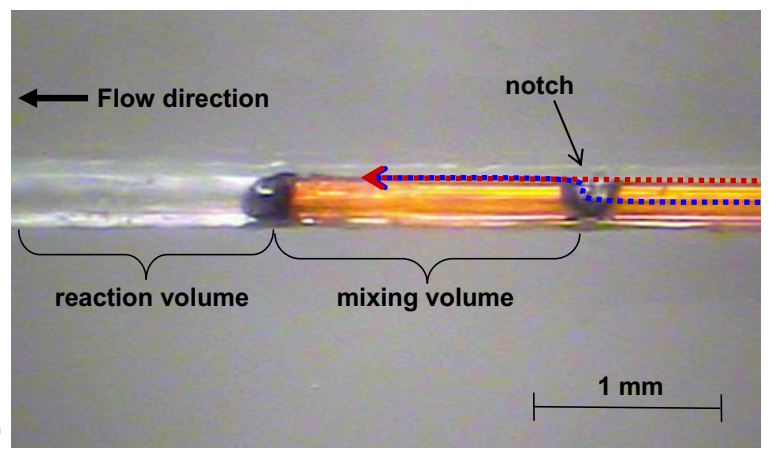

Figure 1. A macroscopic view of the reactor is shown in (a). The channels (white) and mixing capillary (orange) have been digitally enhanced for clarity. The main channel runs the length of the device and accommodates the $153 \mu \mathrm{m}$ o.d. fused silica 'mixing capillary'. A second channel delivers fluid into the main channel and around the mixing capillary. (b) A microscopic view of the distal end of the mixing capillary within the main channel. Blue and red arrows indicate the flow of reactants before and during mixing. The total volume of the mixing region is $\sim 10 \mathrm{~nL}$. Moving the mixing capillary within the main channel changes the reaction volume and hence the delay between mixing and ESI.

200-250 V). In a typical kinetic run, the distal end of the mixing capillary was initially positioned at the ESI outlet of the main microfluidic channel. The solution passing through the mixing capillary was $10 \mu \mathrm{M}$ cytochrome $c$ in Ultrapure water. The solution passing around the mixing capillary was $10 \%$ (vol/vol) acetic acid in Ultrapure water. Both solutions were injected at a rate of $7 \mu \mathrm{L} / \mathrm{min}$. Kinetics were measured by withdrawing the mixing capillary at a rate of $2.0 \times 10^{-4} \mathrm{~m} / \mathrm{s}$ such that the reaction volume (the volume between the end of the mixing capillary and the ESI outlet) increased at a rate of $0.3 \mu \mathrm{L} / \mathrm{min}$. As the mixing capillary was withdrawn, full ESI mass spectra were acquired at a rate of $1 \mathrm{~s}^{-1}$. This corresponds to a mass spectrum every $20 \mathrm{~ms}$ of reaction time. However, the relationship between delay volume and analyte age is complicated by laminar flow as discussed in the following section.

\section{Theory and Data Analysis}

\section{Laminar Flow in a Square Channel}

At ESI-compatible flow rates, flow through microfluidic channels is laminar. Square channel laminar flow is characterized by a 3D parabolic velocity profile. The 
general expression for the velocity profile in a rectangular channel is given by [18]:

$$
v(x, y)=v_{\max }\left(1-\left(\frac{|x|}{X}\right)^{n_{x}}\right)\left(1-\left(\frac{|y|}{Y}\right)^{n_{y}}\right)
$$

Equation 1 uses a Cartesian coordinate system whose origin is at the center of the channel. $X$ and $Y$ are the distances from the center of the channel to the wall in the width and height dimensions, respectively (i.e., $X=[1 / 2]$ channel width, $Y=[1 / 2]$ channel height). The coordinates $x$ and $y$ can therefore have values in the ranges $-X \ldots X$ and $-Y \ldots Y$. The exponents $n_{x}$ and $n_{y}$ are calculated from the aspect ratio of the channel, $\alpha=$ height/width. For $\alpha \geq$ [1/3], $n_{x}=2+$ $0.3(\alpha-[1 / 3])$ and $n_{y}=1.7+0.5 \alpha^{-1.4}$ [18]. In the present case, the channel is square, such that $n_{x}=$ $n_{y}=2.2$. The maximum flow velocity $v_{\max }$ can be calculated from the average flow velocity $v_{\text {avg }}$ by:

$$
v_{\max }=v_{a v g}\left(\frac{n_{x}+1}{n_{x}}\right)\left(\frac{n_{y}+1}{n_{y}}\right)
$$

The laminar flow velocity profile convolutes our kinetic data; analytes occupying the center of the channel will be 'younger' when they arrive at the ESI source than analytes near the channel walls. To account for this, we can derive a probability distribution function $P(a)$ that gives the probability of detecting an analyte of age $a$ for a given reaction channel length $l$. Combining $P(a)$ with an expression for analyte concentration as a function of age $C(a)$ and integrating from $a=l / v_{\max }$ to $a=\infty$, gives the average concentration of analyte at the ESI outlet $C_{a v g}$, which is assumed to be proportional to the ESI signal intensity [19]:

$$
C_{a v g}=\int_{v_{\max }}^{\infty} P(a) C(a) d a
$$

This approach for analyzing kinetic data acquired under laminar flow conditions was introduced by Konermann in 1999 [19]. However, in that work $P(a)$ was derived for a circular geometry. In a circular tube, the laminar flow velocity profile is radially symmetric and therefore dependent on a single geometric parameter $r$ (distance from the tube center). In a rectangular channel, the velocity profile is a function of both $x$ and $y$. We must therefore use a different approach to calculate $P(a)$.

For a given $l$, we can define a function $G\left(a_{0}\right)$ that gives the total number of particles with velocity $v \geq l / a_{0}$ (age $a \leq a_{0}$ ) passing through the detector per unit time:

$$
G\left(a_{0}\right)=\rho \int_{-y_{b}}^{y_{b}} \int_{-x_{b}}^{x_{b}} v(x, y) d x d y
$$

The constant $\rho$ represents an analyte concentration factor. To simplify the integral, we can take advantage of the $x$ and $y$ axis symmetry of $v(x, y)$ and restrict our derivation to the $+x,+y$ quadrant. The range of $x$ and $y$ is thereby constrained to $x=0 . X$ and $y=0 . Y$ and the expression is multiplied by 4 . An additional simplification for square channels is that $\mathrm{X}=\mathrm{Y}$ and $n_{x}=n_{y}$. Incorporating these simplifications, and using the condition $v \geq l / a_{0}$, the upper limits of integration are:

$$
\begin{aligned}
& y_{b}=Y\left(1-\frac{l}{a_{0} v_{\max }}\right)^{\frac{1}{n}} \\
& x_{b}=Y\left(1-\frac{l}{a_{0} v_{\max }\left(1-\left(\frac{y}{Y}\right)^{n}\right)}\right)^{\frac{1}{n}}
\end{aligned}
$$

And expression 4 becomes:

$$
G\left(a_{0}\right)=4 Y \rho v_{\max } \int_{y=0}^{y_{b}} \int_{x=0}^{x_{b}}\left(1-\left(\frac{x}{Y}\right)^{n}\right)\left(1-\left(\frac{y}{Y}\right)^{n}\right) d x d y
$$

Using the limits $y_{b}$ and $x_{b}$, expression 6 defines a cross sectional area of $v(x, y)$ bounded by the velocity contour $v=l / a_{0}$. The expression can be integrated analytically in one dimension, but the resulting integral must be solved numerically for most cases of $a_{0}$.

From expression 6, it is evident that the fraction of analyte passing through the detector within the area bounded by $G\left(a_{0}\right)$ is $G\left(a_{0}\right) / G\left(a_{0}=\infty\right)$. This fraction can also be defined as the probability of detecting an analyte with age $a \leq a_{0}$. It follows, therefore, that the probability of detecting an analyte with a particular age, $P(a)$ is:

$$
P(a)=\frac{G\left(a_{0}\right)-G\left(a_{0}-\Delta a\right)}{G\left(a_{\infty}\right)}
$$

where $\Delta \mathrm{a}$ is the width of the age interval. Note that calculations of $\mathrm{P}(\mathrm{a})$ become more accurate as $\Delta \mathrm{a} \rightarrow 0$. Figure 2 plots $P(a)$ for several reaction channel lengths. $P(a)$ can now be substituted into eq 3 for 'square channel laminar flow corrected' analysis of the kinetic data. This derivation of $P(a)$ is more general than those developed previously. It can be used for any channel geometry provided that there is at least one axis of symmetry.

\section{Other Considerations}

The analytical framework introduced above applies only to a fully realized laminar flow. Laminar flow develops over an entrance length $l_{\text {ent }}$ that is easily approximated for a rectangular geometry; $l_{\text {ent }} \approx 2.4 A / P$ where $A$ and $P$ are the area and perimeter the perimeter of the microfluidic channel [20]. In the present study, $l_{\text {ent }}=4.5 \times 10^{-5} \mathrm{~m}$, less than $1 / 5$ the shortest $l$ used $\left(2.0 \times 10^{-4} \mathrm{~m}\right)$. We therefore consider the effects of $l_{\text {ent }}$ on the kinetic data to be 


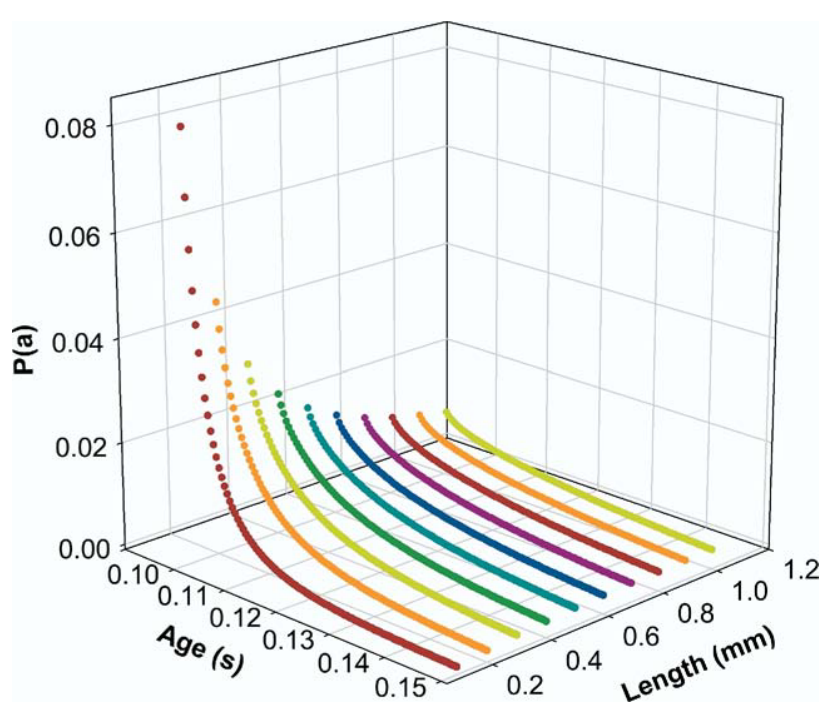

Figure 2. A waterfall plot of $P(a, l)$, giving the probability of detecting particles of particular ages at various reaction channel lengths. In expression $3, P(a)$ is combined with a function describing changing concentration as a function of age due to the reaction, $C(a)$, to give a laminar flow corrected time-dependent ESI signal intensities.

negligible. Data analysis might also be convoluted by Taylor dispersion-based distortion of the laminar flow velocity profile. However, on the scale of the reaction channel $(155 \mu \mathrm{m} \times 155 \mu \mathrm{m})$, significant distortion of the laminar flow profile occurs about seconds [3], much longer than the timescale of our kinetic experiments (final timepoint $=0.65 \mathrm{~s}$ ). The effects of Taylor dispersion can therefore also be neglected.

\section{Results and Discussion}

\section{Fabrication}

One of the aims of this work was to devise fast, simple, and reproducible protocol for ECMD fabrication. Conventional approaches for the fabrication of ECMDs include photolithography $[21,22]$ and polymer imprint techniques [23, 24]. Elegant, highly detailed microscale features can be generated using these methods, but they require specialized facilities, equipment, and expertise. While efforts are being made to simplify photolithographic and polymer imprint fabrication [22], most protocols still require days to go from design to completion. In the present study, microchannels and the microfluidic mixer were formed by laser ablation using a VersaLaser engraving device.

Laser ablation has some disadvantages relative to other microfabrication techniques. First, the generation of ultrafine features is limited by the dot size of the laser and thermal conduction causing melting around the laser dot. Equipped with high-resolution optics, the engraving device used in the present study can generate a dot size of $\sim 15 \mu \mathrm{m}$, which, in PMMA, corresponds to a minimum feature size of $\sim 30 \mu \mathrm{m}$. Since the microfluidic reactor is composed of features that are much larger than this $(155 \mu \mathrm{m})$, the standard resolution optics were sufficient. Second, microfluidic channels generated by laser ablation are not perfectly rectangular, but rounded at their base. The extent of this effect depends on a number of factors including the quality of laser and optics used, but always increases with the aspect ratio $\alpha$. We found that the VersaLaser could generate an essentially rectangular channel in PMMA up to $\alpha \approx 1.3$, which is well above our requirements $(\alpha=1)$.

The main advantage of our fabrication approach is that it is exceedingly straightforward. In this study, designs were generated using widely available illustration software (CorelDraw X3) and then simply 'printed' onto the PMMA substrate. The fabrication process therefore requires no specialized expertise or facility. The production cycle is also quite rapid. Prototypes of the microfluidic reactor generally required $<2.5 \mathrm{~h}$ from design to completion.

\section{Mixing}

In time-resolved experiments, mixing is almost always the main contributor to dead-time. The design of efficient mixers is a major branch of microfluidics research [25-27]. Approaches such as hydrodynamic focusing or the generation of chaotic flow can result in extremely efficient mixing on the micro-scale [25, 27, 28]. The microfluidic reactor introduced in the present study incorporates a mixer that is integrated into the distal end of the mixing capillary (Figure $1 \mathrm{~b}$ ). The mixer operates by forcing reactant solutions to flow together through the narrow space between the mixing capillary and the channel walls. Since this space is defined by a round capillary tightly fit into a square channel, the minimum mixing efficiency occurs at the corners where the distance between the capillary and the channel walls is $\sim 65 \mu \mathrm{m}$. For a mixer length of $2 \mathrm{~mm}$, the total volume of the mixing region is $\sim 10 \mathrm{~nL}$. This corresponds to an average mixing time of $\sim 43 \mathrm{~ms}$ in our experiments.

Given the complications of flow within the mixer (i.e., complex geometry, a developing laminar flow profile, etc.) it is not feasible to devise a theoretical model to predict mixing efficiency. However, an estimate of diffusion length, $L_{\mathrm{D}}=(4 D t)^{1 / 2}$ where $D$ is the diffusion coefficient of protons in bulk water $(\sim 1 \times$ $10^{-8} \mathrm{~m}^{2} \mathrm{~s}^{-1}$ [29], gives an average distance of $41.5 \mu \mathrm{m}$ in $43 \mathrm{~ms}$. Therefore, assuming that the laminar flow layer containing excess protons occupies half of the distance from the capillary to the channel wall, it is not unreasonable to expect complete equilibration of proton concentration within the mixer based on diffusion alone. Furthermore, massive rearrangement of the laminar flow profile upon the incorporation of fluid exiting the notch may significantly enhance mixing efficiency [30].

Mixing efficiency was gauged experimentally by changing the length of the mixer and monitoring the effects on the measured cytochrome $c$ unfolding kinetics. It was observed that when the notch was positioned between 0.5 and $1.5 \mathrm{~mm}$ from the end of the mixing 
capillary, a fraction of the folded protein population did not undergo rapid unfolding but, rather, decreased slowly and linearly as a function of time (data not shown). Both the magnitude of this persistent folded population and the rate at which it was eliminated were dependent on mixer length. This is exactly the result expected if mixing is incomplete upon transfer to the reaction channel. Unmixed fluid passing into the reaction channel will form a laminar flow layer within which native conditions persist. This layer will slowly be eliminated via inefficient diffusive mixing with surrounding flow layers. Ultimately, it was determined that a mixer length of $2 \mathrm{~mm}$ was required for complete mixing before transfer into the reaction channel.

\section{On-Chip Electrospray and Instrument Dead-Time}

The two main considerations for TR ESI-MS experiments with respect to electrospray performance are Taylor cone volume and stability. Taylor cone volume is especially significant because it contributes to the deadtime of the experiment. Taylor cones generated 'onchip' tend to be larger than those produced at conventional capillary outlets due to interactions between the eluent and the edge of the substrate [31]. In this respect, the use of PMMA, which is quite hydrophilic, is disadvantageous. In the present study, an effort was made to reduce spreading by tapering the ESI outlet to a sharp point. In addition, source potentials were at least $300 \mathrm{~V}$ higher than the minimum required to generate a stable Taylor cone. From scaled pictures taken on a CCD camera (Figure 3a), and assuming a radially symmetric shape, Taylor cone volumes were estimated to be in the range of $1-3 \mathrm{~nL}$, corresponding to $4-12 \mathrm{~ms}$ of dead-time at $14 \mu \mathrm{L} / \mathrm{min}$. The dead-time of the microfluidic reactor was therefore $43 \mathrm{~ms}$ (mixing) $+12 \mathrm{~ms}$ (Taylor cone) + $1 \mathrm{~ms}$ (desolvation [32] $\approx 56 \mathrm{~ms}$.

Taylor cone stability is the dominant factor affecting variability in ESI signal intensity. In TR ESI-MS experiments, Taylor cone instability results in noisy and in extreme cases un-interpretable kinetic data. Signal stability for ECMDs with 'on-chip' ESI is generally lower than that of capillary-based devices and the present microfluidic reactor is no exception. Figure $3 \mathrm{~b}$ shows a typical TIC of $5 \mu \mathrm{M}$ cytochrome $c$ in $5 \%$ (vol/vol) acetic acid at a reaction time of 250 ms (roughly halfway through the unfolding process). Over $5 \mathrm{~min}$ of data collection, the standard deviation for TIC signal intensity was 5219 or $2.8 \%$. This degree of signal stability was more than sufficient to provide reliable kinetic data.

\section{Cytochrome c Unfolding}

Cytochrome $c$ is a small heme protein from the inner membrane of the mitochondrion. In comparison to other proteins, the biophysical properties of cytochrome $c$ have been extensively characterized [33]. Cytochrome $c$ is highly amenable to ESI, making it a convenient test subject for new MS-based analytical approaches. The
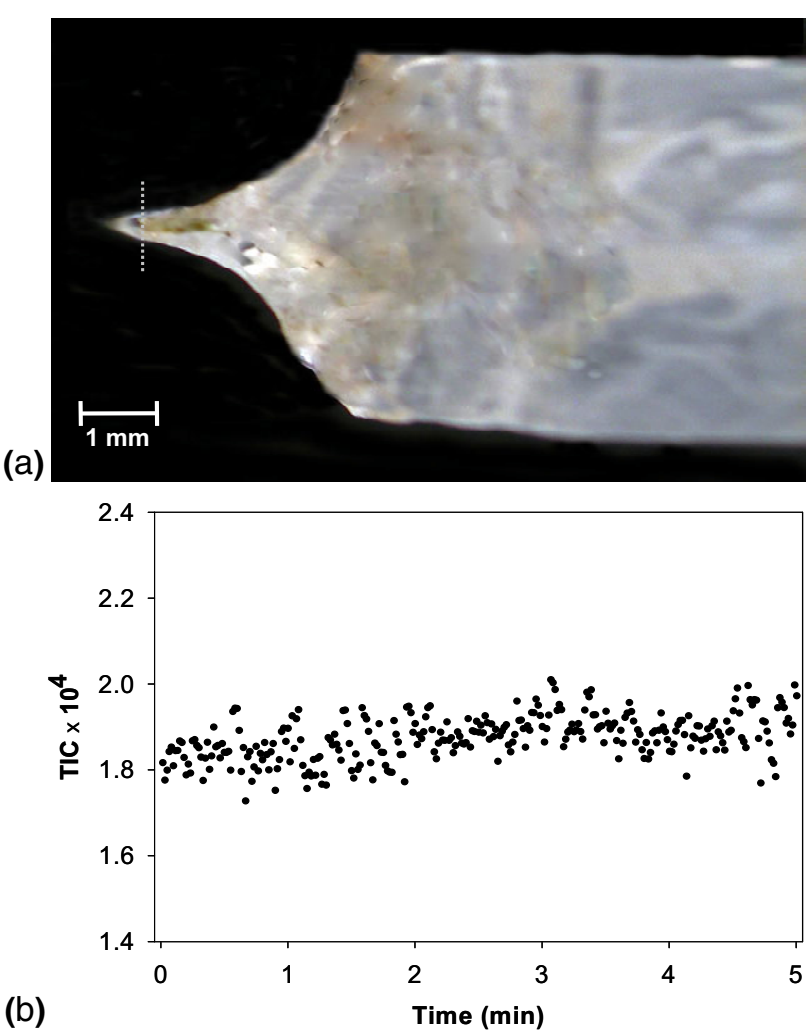

Figure 3. (a) Representative image from a series of images used to estimate Taylor cone volume. The grey dotted line indicates the edge of the chip. The ESI outlet of the device was $\sim 4 \mathrm{~mm}$ from the curtain plate, which was held at $-1000 \mathrm{~V}$. Source potential was $+5500 \mathrm{~V}$. A stable Taylor cone was observed at a source potential of $+5250 \mathrm{~V}$ under these conditions. (b) The TIC for a $5 \mu \mathrm{M}$ solution of cytochrome $c$ in $5 \%$ acetic acid. The mixing capillary was positioned to allow $250 \mathrm{~ms}$ of reaction time. Source conditions were the same as (a). The signal has a standard deviation of 5219 or $2.8 \%$.

folding kinetics of cytochrome $c$ are well studied [34] and were among the first processes to be investigated by time-resolved ESI-MS [6]. Kinetic cytochrome $c$ unfolding studies are less common, possibly due to the assumption that transient intermediates will not become populated under strongly denaturing conditions and that therefore unfolding will be mechanistically uninteresting [35]. Kinetic unfolding intermediates of cytochrome $c$ have been detected in optical studies, however [36].

Acid induced cytochrome $c$ unfolding was used to gauge the effectiveness of our microfluidic reactor for monitoring millisecond time-scale kinetics. The reactor was first operated in 'spectral mode' in which the mixer is fixed at various positions within the main channel. Spectral mode allows the reaction to be monitored indefinitely at fixed time-points, facilitating the acquisition of high signal-to-noise mass spectra. Figure 4 shows mass spectra corresponding to cytochrome $c$ unfolding at $70 \mathrm{~ms}$ (a), $200 \mathrm{~ms} \mathrm{(b)} \mathrm{and} 650 \mathrm{~ms}$ (c). Between $70 \mathrm{~ms}$ and $200 \mathrm{~ms}$, there is a shift in the dominant charge state from $8+$ to $9+$, suggesting the involvement of a partially unfolded, 'native-like' intermediate in the unfolding process. The transition from the intermediate to the unfolded protein appears to occur in a single step. 


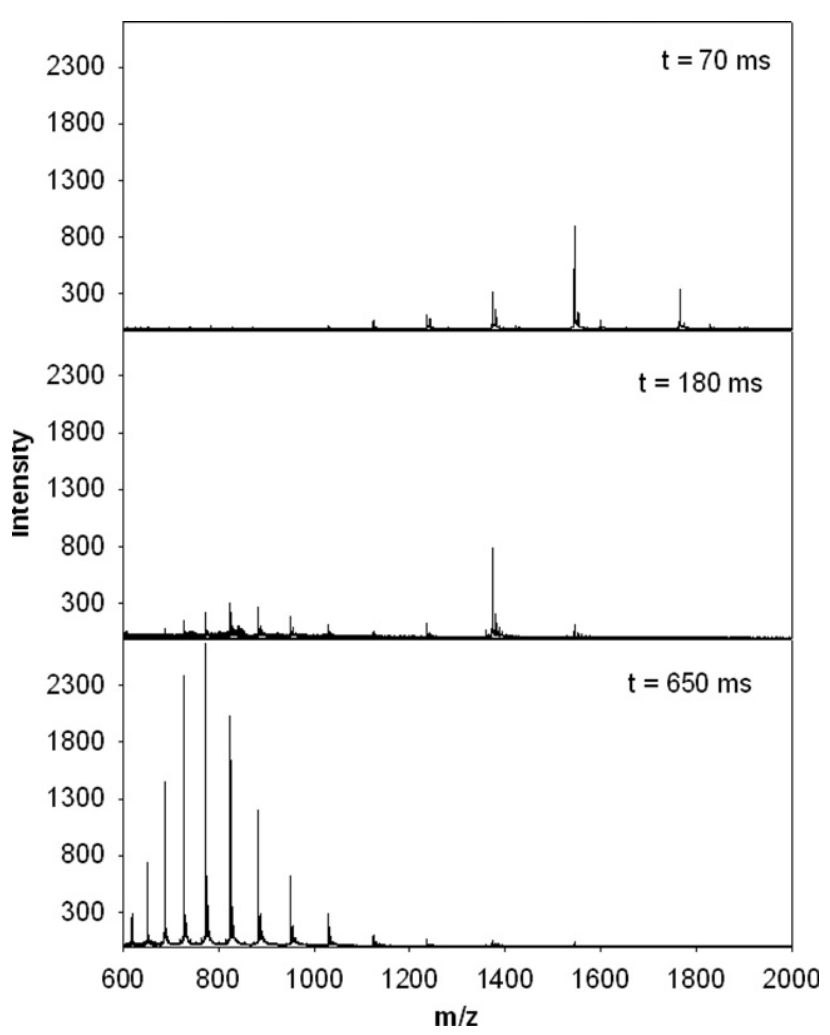

Figure 4. TR ESI mass spectra of kinetic cytochrome $c$ unfolding at $70 \mathrm{~ms}, 200 \mathrm{~ms}$, and $650 \mathrm{~ms}$. Conditions after mixing were $5 \mu \mathrm{M}$ cytochrome $c$ in $5 \%(\mathrm{vol} / \mathrm{vol})$ acetic acid. A rapid transition was observed from a folded form of the protein with dominant charge state $8+$ to an unfolding intermediate with dominant charge state $9+$. The transition from $9+$ to a broad distribution centered on $16+$ occurred over a longer time-frame.

A more detailed analysis can be carried out in 'kinetics mode' where the mixing capillary is steadily withdrawn while mass spectra are collected. Figure 5 shows the results of a typical kinetics mode experiment. Each trace represents the ion current for peaks corresponding to various charge states of cytochrome $c$ from $7+$ to $19+$. The observed kinetics can be roughly subdivided into three categories: Charge states $7+$ and $8+$ undergo monophasic exponential decay. Charge states $9+$ and $10+$ exhibit classic intermediate kinetics, i.e., an exponential rise followed by an exponential decay. The intensities of charge states $11+$ to $18+$ increase exponentially after a brief delay.

The data were fit to expression 3 using a FORTRAN program developed previously [3] adjusted for square channel laminar flow as described in theory section. Fits are shown as solid lines in Figure 5. The data were analyzed using an implicit global analysis strategy based on that in reference [14]. The concentration versus time dependences for each cytochrome $c$ charge state, $C(a, m / z)$, were assumed to correspond to a sum of exponentials plus an offset. It was found that two exponentials were sufficient to obtain satisfactory fits to all of the kinetic data. The first exponential, associated with the formation of the intermediate, had a rate constant $k_{1 o b s}=32 \mathrm{~s}^{-1} \pm 5$. This is in agreement with stopped-flow fluorescence data acquired by Bhuyan et al. [36] in $3.1 \mathrm{M} \mathrm{GdnHCl}$, although it is difficult to make a direct comparison given the significantly different conditions employed in that study. The second exponential is associated with the transition from the intermediate to the 'unfolded' protein. This process was found to occur at $k_{2 o b s}=7.5 \mathrm{~s}^{-1} \pm 0.1$. This rate agrees with the slow unfolding phase observed by Bhuyan et al. by stopped flow far UV-CD [36].

While folding intermediates are quite common $[13,37,38]$, unfolding intermediates are less commonly detected, particularly in a small proteins $[14,35]$. Cytochrome $c$ may be an exception due to its covalently bound heme which could act as a structural nucleus during unfolding. Alternatively, the complex unfolding landscapes predicted by some computer models [39] and observed for large proteins [14] may apply equally to small proteins. The microfluidic reactor introduced here can help to shed light on this question by facilitating the detection of transient unfolding intermediates, especially those that cannot be observed by conventional optical methods. However, the current charge state distribution-based analysis provides little information as to the nature of those intermediates. More detailed characterizations will be possible with the inclusion of additional microfluidic modules for $\mathrm{H} / \mathrm{D}$ exchange pulse labeling and on-chip proteolytic digestion.

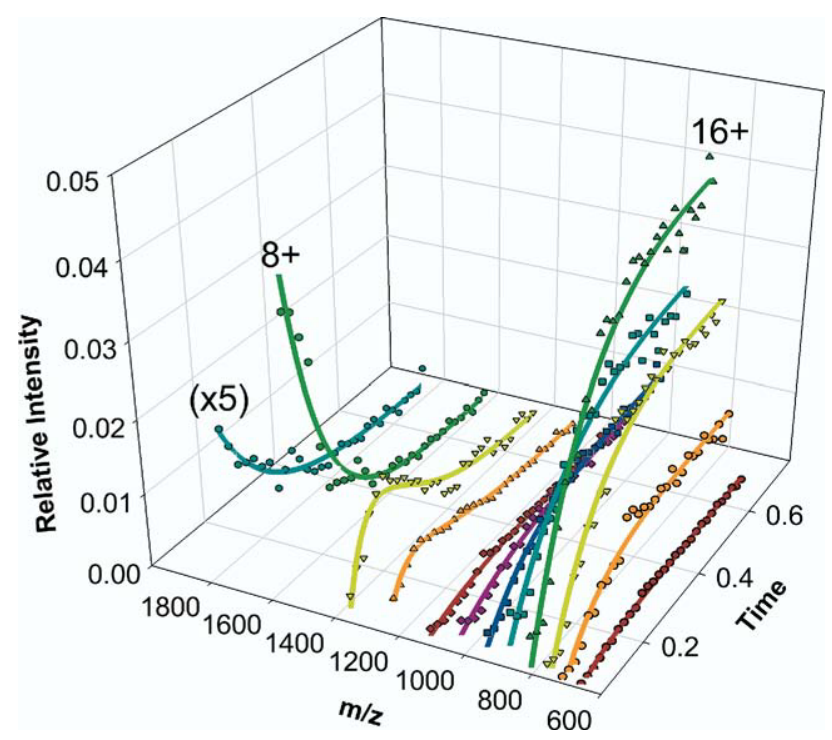

Figure 5. A global view of cytochrome $c$ unfolding kinetics. Charge state $7+$ intensities have been multiplied by a factor of 5 to improve visibility. Charge states which do not provide reliable kinetics due to low signal-to-noise are excluded. Solid lines represent fits to the data using expression 3. $P(a)$ was calculated as described in the theory section. In keeping with a global analysis strategy described previously [14], $C(a)$ was assumed to have the form of a linear combination of exponentials plus an offset. Satisfactory fits to all of the kinetic data were acquired using two exponentials with rate constants $k_{1 \mathrm{obs}}=32 \mathrm{~s}^{-1} \pm 5$ and $k_{2 \mathrm{obs}}=7.5$ $\pm 0.1 \mathrm{~s}^{-1}$ 


\section{Conclusions}

While the device introduced in the present study is suitable for a broad range of applications, there are a number of improvements that will be pursued in future work. One major issue is the significant mixing time, which results primarily from the accommodation of a round capillary into a square channel. We are currently redesigning the device to incorporate square capillaries, which have recently become commercially available. Since the 'square in a square' geometry will also improve mixing efficiency, mixing dead-times may be reduced to below the contribution from Taylor cone volume. Additional design changes are underway to reduce Taylor cone volume and increase Taylor cone stability by using thinner substrates and optimizing the tapering method. Design improvements are being undertaken in tandem with the development of multimodule ECMDs. Applications include measuring enzyme kinetics under native salt conditions and detailed characterizations of transient species in protein folding and enzyme reactions.

\section{Acknowledgments}

The authors thank Neal Madras, Hanna Jankowski, and Jane Heffernan for helpful discussions. The authors gratefully acknowledge funding by the Canadian Foundation for Innovation.

\section{References}

1. Erickson, D.; Li, D. Q. Integrated Microfluidic Devices. Anal. Chim. Acta 2004, 507, 11-26

2. Lion, N.; Rohner, T. C.; Dayon, L.; Arnaud, I. L.; Damoc, E.; Youhnovski, N.; Wu, Z. Y.; Roussel, C.; Josserand, J.; Jensen, H.; Rossier, J. S.; Przybylski, M.; Girault, H. H. Microfluidic Systems in Proteomics. Electrophoresis 2003, 24, 3533-3562.

3. Wilson, D. J.; Konermann, L. A Capillary Mixer with Adjustable Reaction Chamber Volume for Millisecond Time-Resolved Studies by Electrospray Mass Spectrometry. Anal. Chem. 2003, 75, 6408-6414.

4. Wilson, D. J.; Konermann, L. Mechanistic Studies on Enzymatic Reactions by Electrospray Ionization MS Using a Capillary Mixer with Adjustable Reaction Chamber Volume for Time-Resolved Measurements. Anal. Chem. 2004, 76, 2537-2543.

5. Simmons, D. A.; Wilson, D. J.; Lajoie, G. A.; Doherty-Kirby, A.; Konermann, L. Subunit Disassembly and Unfolding Kinetics of Hemoglobin Studied by Time-Resolved Electrospray Mass Spectrometry. Biochemistry 2004, 43, 14792-14801.

6. Konermann, L.; Collings, B. A.; Douglas, D. J. Cytochrome $c$ Folding Kinetics Studied by Time-Resolved Electrospray Ionization Mass Spectrometry. Biochemistry 1997, 36, 5554-5559.

7. David, L.; Zechel, L. K.; Stephen, G.; Withers, S. G.; Douglas, D. J. Pre-Steady State Kinetic Analysis of an Enzymatic Reaction Monitored by Time-Resolved Electrospray Ionization Mass Spectrometry. Biochemistry 1998, 37, 7664-7669.

8. Simmons, D. A.; Konermann, L. Characterization of Transient Protein Folding Intermediates during Myoglobin Reconstitution by TimeResolved Electrospray Mass Spectrometry with On-Line Isotopic Pulse Labeling. Biochemistry 2002, 41, 1906-1914.

9. Kolakowski, B. M.; Konermann, L. From Small-Molecule Reactions to Protein Folding: Studying Biochemical Kinetics by Stopped-Flow Electrospray Mass Spectrometry. Anal. Biochem. 2001, 292, 107-114.

10. Kolakowski, B. M.; Simmons, D. A.; Konermann, L. Stopped-Flow Electrospray Ionization Mass Spectrometry: A New Method for Studying Chemical Reaction Kinetics in Solution. Rapid Commun. Mass Spectrom. 2000, 14, 772-776.

11. Wolf-Watz, M.; Thai, V.; Henzler-Wildman, K.; Hadjipavlou, G.; Eisenmesser, E. Z.; Kern D. Linkage Between Dynamics and Catalysis in a Thermophilic-Mesophilic Enzyme Pair. Nat. Struct. Mol. Biol. 2004, 11, 945-949.
12. Krishna, M. M. G.; Maity, H.; Rumbley, J. N.; Lin, Y.; Englander, S. W. Order of Steps in the Cytochrome $c$ Folding Pathway: Evidence for a Sequential Stabilization Mechanism. J. Mol. Biol. 2006, 359, 1410-1419.

13. Raschke, T. M.; Marqusee, S. The kinetic Folding Intermediate of Ribonuclease $\mathrm{H}$ Resembles the Acid Molted Globule and Partially Unfolded Molecules Detected Under Native Conditions. Nat. Struct. Biol. 1997, 4, 505-505.

14. Wilson, D. J.; Rafferty, S. P.; Konermann, L. Kinetic Unfolding Mechanism of the Inducible Nitric Oxide Synthase Oxygenase Domain Determined by Time-Resolved Electrospray Mass Spectrometry. Biochemistry 2005, 44, 2276-2283.

15. Pan, J.; Wilson, D. J.; Konermann, L. Pulsed Hydrogen Exchange and Electrospray Charge-State Distribution as Complementary Probes of Protein Structure in Kinetic Experiments: Implications for Ubiquitin Folding. Biochemistry 2005, 44, 8627-8633.

16. Pan, J.; Rintala-Dempsey, A. C.; Li, Y.; Shaw, G. S.; Konermann, L. Folding Kinetics of the S100A11 Protein Dimer Studied by TimeResolved Electrospray Mass Spectrometry and Pulsed HydrogenDeuterium Exchange. Biochemistry 2006, 45, 3005-3013.

17. Konermann, L.; Pan, J.; Wilson, D. J. Protein Folding Mechanisms Studied by Time-resolved Electrospray Mass Spectrometry. BioTechniques 2006, 40, 134-136.

18. Ebadian, M. A.; Dong, Z. F. Forced Convection, Internal Flow in Ducts, In Handbook of Heat Transfer 3rd ed., Rohsenow, W. M., Hartnett, J. P. Cho, Y. I., Eds., McGraw Hill: New York, 1998.

19. Konermann, L. Monitoring Reaction Kinetics in Solution by ContinuousFlow Methods: The Effects of Convection and Molecular Diffusion Under Laminar Flow Conditions. J. Phys. Chem. A 1999, 103, 7210-7216.

20. Wilson, D. J.; Konermann, L. Ultrarapid Desalting of Protein Solutions for Electrospray Mass Spectrometry in a Microchannel Laminar Flow Device. Anal. Chem. 2005, 77, 6887-6894.

21. Schultz, G. A.; Corso, T. N.; Prosser, S. J.; Zhang, S. A Fully Integrated Monolithic Microchip Electrospray Device for Mass Spectrometry. Anal. Chem. 2000, 72, 4058-4063

22. Freire, S. L. S.; Yang, H.; Wheeler, A. R. A Practical Interface for Microfluidics and Nanoelectrospray Mass Spectrometry. Electrophoresis 2008, 29, 1836-1843.

23. Bedair, M. F.; Oleschuk, R. D. Fabrication of Porous Polymer Monoliths in Polymeric Microfluidic Chips as an Electrospray Emitter for Direct Coupling to Mass Spectrometry. Anal. Chem. 2006, 78, 1130-1138.

24. Mills, C. A.; Martinez, E.; Bessueille, F.; Villanueva, G.; Bausells, J.; Samitier, J.; Errachid, A. Production of Structures for Microfluidics Using Polymer Imprint Techniques. Microelectron. Eng. 2005, 78-79, 695-700.

25. Xia, H. M.; Wan, S. Y. M.; Shu, C.; Chew, Y. T. Chaotic Micromixers Using Two-Layer Crossing Channels to Exhibit Fast Mixing at Low Reynolds Numbers. Lab. Chip. 2005, 5, 748-755.

26. Stone, H. A.; Stroock, A. D.; Ajdari, A. Engineering Flows in Small Devices: Microfluidics Toward a Lab-on-a-Chip. Ann. Rev. Fluid Mech. 2004, 36, 381-411.

27. Ottino, J. M.; Wiggins, S. Introduction: Mixing in Microfluidics. Philosoph. Trans. Roy. Soc. 2004, 362, 923-935.

28. Abonnenc, M.; Dayon, L.; Perruche, B.; Lion, N.; Girault, H. H. Electrospray Micromixer Chip for On-Line Derivatization and Kinetic Studies. Anal. Chem. 2008, 80, 3372-3378.

29. Georgievskii, Y.; Medvedev, E. S.; Stuchebrukhov, A. A. Proton Transport Via the Membrane Surface. Biophys. J. 2002, 82, 2833-2846.

30. Yamaguchi, Y.; Takagi, F.; Watari, T.; Yamashita, K.; Nakamura, H.; Shimizu, H.; Maeda, H. Interface Configuration of the Two Layered Laminar Flow in a Curved Microchannel. Chem. Eng. J. 2004, 101, 367-372.

31. Xue, Q. F.; Foret, F.; Dunayevskiy, Y. M.; Zavracky, P. M.; McGruer N. E.; Karger, B. L. Multichannel Microchip Electrospray Mass Spectrometry. Anal. Chem. 1997, 69, 426-430.

32. Kebarle, P. A Brief Overview of the Present Status of the Mechanisms Involved in Electrospray Mass Spectrometry. J. Mass Spectrom. 2000, 35, 804-817.

33. Keilin, D.; Hartree, E. F. Preparation of Pure cytochrome $c$ from Heart Muscle and Some of Its Properties. P. Roy. Soc. B, Biol. Sci. 1937, 122, 298-308.

34. Winkler, J. R. Cytochrome $c$ folding dynamics. Curr. Opin. Struct. Biol. 2004, 8, 169-174.

35. Juneja, J.; Udgaonkar, J. B. Characterization of the Unfolding of Ribonuclease a by a Pulsed Hydrogen Exchange Study: Evidence for Competing Pathways for Unfolding. Biochemistry 2002, 41, 2641-2654.

36. Bhuyan, A. K.; Udgaonkar, J. B. Multiple Kinetic Intermediates Accumulate During the Unfolding of Horse Cytochrome $c$ in the Oxidized State. Biochemistry 1998, 37, 9147-9155.

37. Bai, Y. W.; Sosnick, T. R.; Mayne, L.; Englander, S. W. Protein Folding Intermediates-Native-State Hydrogen-Exchange. Science 1995, 269, 192 197.

38. Englander, S. W. Protein Folding Intermediates and Pathways Studied by Hydrogen Exchange. Ann. Rev. Biophys. Biomol. Struct. 2000, 29, 213-238.

39. Lazaridis, T.; Karplus, M. "New View" of Protein Folding Reconciled with the Old Through Multiple Unfolding Simulations. Science. 1997, 278, 1928-1931. 